\title{
Kernel Computation in Morphological Bidirectional Associative Memories
}

\author{
Gonzalo Urcid $^{1, \star}$, Gerhard X. Ritter ${ }^{2}$, and Laurentiu Iancu ${ }^{2}$ \\ 1 Optics Department, INAOE, Tonantzintla, Pue. 72000, Mexico \\ gurcid@inaoep.mx \\ 2 CISE Department, University of Florida, Gainesville, FL 32611-6120, USA \\ \{ritter, liancu\}@cise.ufl.edu
}

\begin{abstract}
Morphological associative memories (MAMs) use a lattice algebra approach to store and recall pattern associations. The lattice matrix operations endow MAMs with properties that are completely different than those of traditional associative memory models. In the present paper, we focus our attention to morphological bidirectional associative memories (MBAMs) capable of storing and recalling non-boolean patterns degraded by random noise. The notions of morphological strong independence (MSI), minimal representations, and kernels are extended to provide the foundation of bidirectional recall when dealing with noisy inputs. For arbitrary pattern associations, we present a practical solution to compute kernels in MBAMs by induced MSI.
\end{abstract}

\section{Introduction}

The foundation of morphological associative memories was established in 9 , where it was proved that morphological auto-associative memories have unlimited storage capacity and provide perfect recall for noncorrupted boolean inputs in comparison with traditional associative memories based on correlation encoding such as the classical Hopfield auto-associative memories [3,6]. Correlation encoding requires that the key vectors are orthogonal in order to exhibit perfect recall of the fundamental associations [14]. The morphological auto-associative memory does not restrict the domain of they key vectors in any way. Thus, as many associations as desired can be encoded into the memory; one step convergence and perfect recall of boolean noisy patterns using the idea of kernels were also settled [9]. Furthermore, the theoretical framework for morphological bidirectional associative memories, developed in [10], showed again, that for some binary pattern classes, MBAMs have large storage capacity and superior bidirectional recall than traditional BAM models [5] and also competitive with other feedforward BAM networks [15]. A characterization of kernel vectors for binary patterns that provided for a direct method for kernel computation as well as bounds for the allowable amount of corruption of the exemplar patterns that guarantee perfect recall appeared in 13. An additional development that

\footnotetext{
* Corresponding author. Fax: +52 (222) 247-2940; Tel.: +52 (222) 266-3100 Ext.8205
} 
uses the notion of dual kernels to enhance the error correction capability of binary auto-associative morphological memories has been introduced in [14. By redefining the notion of kernels, together with new concepts such as morphological strong independence and minimal representations of exemplar non-boolean patterns, MAMs were shown to be robust in the presence of noise [11]12.

Our work is organized as follows: Section 2 gives a brief background of lattice matrix operations for dealing with MAMs, and Section 3 provides an overview of the main results obtained from previous research on MAMs, where greyscale image pattern associations are used to illustrate their performance. Section 4 presents the known theoretical results related to MBAMs for non-boolean patterns including the kernel methodology for storing and recalling associations based on the notions of morphological strong independence (MSI) and minimal representations. Section 5 presents a new procedure for the computation of kernels by induced MSI. Finally, in Section 6 we give our conclusion to the research presented here.

\section{Lattice Matrix Algebra}

Lattice matrix operations are defined componentwise using the binary operations of the bounded lattice-group algebraic structure of $\mathbb{R}_{ \pm \infty}=\mathbb{R} \cup\{-\infty,+\infty\}$ [2] . The binary operators for the maximum or minimum of two numbers are denoted with the "join" and "meet" symbols employed in lattice theory, i.e., $x \vee y=$ $\max (x, y)$ and $x \wedge y=\min (x, y)$. For example, the maximum of two matrices $A, B$ of the same size $m \times n$ is defined as $(A \vee B)_{i j}=a_{i j} \vee b_{i j}$, for all $i=1, \ldots, m$ and $j=1, \ldots, n$. Inequalities between matrices are also verified elementwise, e. g., $A \leq B$ if and only if $a_{i j} \leq b_{i j}$. On the other hand, the conjugate matrix $A^{*}$ is defined as $-A^{t}$ where $A^{t}$ denotes usual matrix transposition, or equivalently, $\left(A^{*}\right)_{i j}=a_{j i}^{*}$, hence $(A \vee B)^{*}=A^{*} \wedge B^{*}$. In addition, for appropriately sized matrices $A, B$, the $i j$ th entry of the max-sum and the min-sum of $A$ and $B$, is defined respectively, for all $i=1, \ldots, m$ and $j=1, \ldots, n$, as follows

$$
(A \nabla B)_{i j}=\bigvee_{k=1}^{p}\left(a_{i k}+b_{k j}\right) \text { and }(A \bowtie B)_{i j}=\bigwedge_{k=1}^{p}\left(a_{i k}+b_{k j}\right)
$$

where, e. g., $\bigwedge_{k=1}^{p} a_{k}$ is the minimum of the set of numbers $\left\{a_{1}, \ldots, a_{p}\right\}$.

The relationship $(A \nabla B)^{*}=B^{*} \square A^{*}$ holds for any $A, B$, and establishes the duality between both types of lattice matrix sums. Finally, the morphological outer sum of two vectors $\boldsymbol{x} \in \mathbb{R}^{n}$ and $\boldsymbol{y} \in \mathbb{R}^{m}$, is given by the $m \times n$ matrix (note that $\boldsymbol{y} \oplus \boldsymbol{x}^{t}=\boldsymbol{y} \nabla \boldsymbol{x}^{t}=\boldsymbol{y} \nabla \boldsymbol{x}^{t}$ )

$$
\boldsymbol{y} \oplus \boldsymbol{x}^{t}=\left(\begin{array}{ccc}
y_{1}+x_{1} & \cdots & y_{1}+x_{n} \\
\vdots & \ddots & \vdots \\
y_{m}+x_{1} & \cdots & y_{m}+x_{n}
\end{array}\right) \text {. }
$$




\section{Morphological Associative Memories}

For a given set of pattern associations $\left\{\left(\boldsymbol{x}^{\xi}, \boldsymbol{y}^{\xi}\right) \in \mathbb{R}^{n} \times \mathbb{R}^{m}: \xi=1, \ldots, k\right\}$ we define a pair of associated pattern matrices $(X, Y)$, where $X=\left(\boldsymbol{x}^{1}, \ldots, \boldsymbol{x}^{k}\right)$ and $Y=\left(\boldsymbol{y}^{1}, \ldots, \boldsymbol{y}^{k}\right)$. Thus, $X$ is of dimension $n \times k$ with $i, j$ th entry $x_{i}^{j}$ and $Y$ is of dimension $m \times k$ with $i, j$ th entry $y_{i}^{j}$. To store $k$ vector pairs $\left(\boldsymbol{x}^{1}, \boldsymbol{y}^{1}\right), \ldots,\left(\boldsymbol{x}^{k}, \boldsymbol{y}^{k}\right)$ in an $m \times n$ MAM we use the morphological outer sum as follows [9]. The minmemory $W_{\mathrm{XY}}$ and the max-memory $M_{\mathrm{XY}}$, that store a set of pattern associations $(X, Y)$ are given, respectively, by the expressions

$$
\begin{array}{ll}
W_{\mathrm{XY}}=Y \bowtie X^{*}=\bigwedge_{\xi=1}^{k}\left[\boldsymbol{y}^{\xi} \oplus\left(-\boldsymbol{x}^{\xi}\right)^{t}\right] & \text { or } w_{i j}=\bigwedge_{\xi=1}^{k}\left(y_{i}^{\xi}-x_{j}^{\xi}\right) \\
M_{\mathrm{XY}}=Y \nabla X^{*}= & \bigvee_{\xi=1}^{k}\left[\boldsymbol{y}^{\xi} \oplus\left(-\boldsymbol{x}^{\xi}\right)^{t}\right] \text { or } m_{i j}=\bigvee_{\xi=1}^{k}\left(y_{i}^{\xi}-x_{j}^{\xi}\right) .
\end{array}
$$

We speak of a hetero-associative morphological memory (HMM) if $X \neq Y$ and an auto-associative morphological memory (AMM) if $X=Y$. From (2), for each $\xi, \boldsymbol{y}^{\xi} \times\left(-\boldsymbol{x}^{\xi}\right)^{t}$ is a matrix $A^{\xi}$ of size $m \times n$ that memorizes the association pair $\left(\boldsymbol{x}^{\xi}, \boldsymbol{y}^{\xi}\right)$, hence $W_{\mathrm{XY}}=\bigwedge_{\xi=1}^{k} A^{\xi}$ and $M_{\mathrm{XY}}=\bigvee_{\xi=1}^{k} A^{\xi}$, which suggests the given names. We use $w_{i j}$ and $m_{i j}$ as an alternative notation for the $i j$ th entries of $W_{\mathrm{XY}}$ and $M_{\mathrm{XY}}$ if there is no confusion about which association is under discussion. Since, $M_{\mathrm{YX}}^{*}=\left(X \nabla Y^{*}\right)^{*}=Y \square X^{*}=W_{\mathrm{XY}}$ and $W_{\mathrm{YX}}^{*}=\left(X \nabla Y^{*}\right)^{*}=Y \nabla X^{*}=$ $M_{\mathrm{XY}}$, the retrieval of pattern $\boldsymbol{y}^{\xi}$ from pattern $\boldsymbol{x}^{\xi}$ can be expressed using the direct memory schemes (the vertical bar means "or"),

$$
\boldsymbol{x}^{\xi} \rightarrow\left\{W_{\mathrm{XY}} \mid M_{\mathrm{XY}}\right\} \rightarrow \boldsymbol{y}^{\xi}
$$

where either one of $W_{\mathrm{XY}}$ or $M_{\mathrm{XY}}$ or their corresponding duals may be used. In a similar fashion, $W_{\mathrm{YX}}=M_{\mathrm{XY}}^{*}$ and $M_{\mathrm{YX}}=W_{\mathrm{XY}}^{*}$, hence recalling the pattern $\boldsymbol{x}^{\xi}$ from pattern $\boldsymbol{y}^{\xi}$ can be realized using the conjugate or reverse memory schemes,

$$
\boldsymbol{y}^{\xi} \rightarrow\left\{W_{\mathrm{YX}} \mid M_{\mathrm{YX}}\right\} \rightarrow \boldsymbol{x}^{\xi}
$$

The conditions of perfect recall for perfect input were established in $[9]$ and we repeat them here for convenience. Specifically, $W_{\mathrm{XY}} \nabla X=Y$ or $M_{\mathrm{XY}} \rrbracket X=Y$, if and only if, for each row index $i \in\{1, \ldots, m\}$ and each pattern index $\gamma \in$ $\{1, \ldots, k\}$, there exists an index $j \in\{1, \ldots, n\}$ which depends on $i, \gamma$, such that

$$
y_{i}^{\gamma}-x_{j}^{\gamma}=w_{i j} \text { or } y_{i}^{\gamma}-x_{j}^{\gamma}=m_{i j}
$$

It is important to remark that, the conditions for perfect recall using MAMs may not be satisfied for arbitrary association pairs $(X, Y)$, with $X \neq Y$, that arise in most practical applications. However, even in the case that for each pattern $\boldsymbol{x}^{\gamma}$ several row indexes do exist for which the expressions in (7) are not satisfied, the memories $W_{\mathrm{XY}}$ and $M_{\mathrm{XY}}$ still provide a storing mechanism 
with almost perfect recall, in the sense of a suitable distance measure, between the original pattern $\boldsymbol{y}_{o}^{\xi}$ and the recalled pattern $\boldsymbol{y}_{r}^{\xi}$. In particular, we use the normalized mean square error (NMSE), denoted by $\varepsilon\left(\boldsymbol{y}_{o}^{\xi}, \boldsymbol{y}_{r}^{\xi}\right)$, to quantify the difference between $\boldsymbol{y}_{o}^{\xi}$ and $\boldsymbol{y}_{r}^{\xi}$, when recalling stored patterns by means of a specific hetero-associative memory scheme. The following example involving nonboolean patterns of high dimensionality illustrates our claim.

Consider the five pattern image associations $\left(\boldsymbol{p}^{1}, \boldsymbol{q}^{1}\right), \ldots,\left(\boldsymbol{p}^{5}, \boldsymbol{q}^{5}\right)$ shown in Fig. (11). Each individual pattern $\boldsymbol{p}^{\xi}$ or $\boldsymbol{q}^{\xi}$ is a $50 \times 50$ pixels 256 -gray scale image. For uncorrupted input, almost perfect recall is obtained if we use either of the memory schemes given by (5) or (6). Using the standard row-scan method, each pattern image, e.g., $\boldsymbol{p}^{\xi}$ can be converted into a pattern vector $\boldsymbol{x}^{\xi}=\left(x_{1}^{\xi}, \ldots, x_{2500}^{\xi}\right) \in \mathbb{R}^{2500}$ of $X$ by defining, $x_{50(r-1)+c}^{\xi}=p^{\xi}(r, c)$ for $r, c=1, \ldots, 50$ (pattern vector $\boldsymbol{q}^{\xi}$ is similarly defined for $\boldsymbol{y}^{\xi}$ of $Y$ ). Figure 2 shows the results when applying the memory scheme of (5) using the canonical memories $W_{\mathrm{XY}}$ and $M_{\mathrm{XY}}$. A visual inspection does not reveal immediately the hidden differences that cause the recall to be non-perfect since $\varepsilon\left(\boldsymbol{y}_{o}^{\xi}, \boldsymbol{y}_{r}^{\xi}\right) \approx 10^{-4}$ for $\xi=1, \ldots, 5$. Although, for a given arbitrary set $(X, Y)$ of pattern associations, the HMMs, $W_{\mathrm{XY}}$ (or $W_{\mathrm{YX}}$ ) and $M_{\mathrm{XY}}\left(\right.$ or $M_{\mathrm{YX}}$ ) are not necessarily perfect recall memories, they still can be applied successfully to deal with noisy inputs.
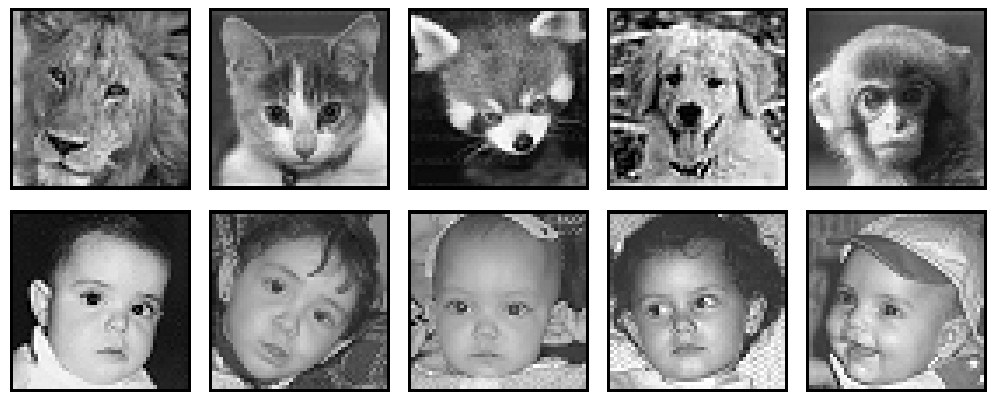

Fig. 1. The association $(X, Y)$ that was used in constructing the memories $W_{\mathrm{XY}}$ and $M_{\mathrm{XY}}$ (of size $\left.2500 \times 2500\right)$. First row: patterns of $X$; second row: patterns of $Y$

\section{MBAMs and the Kernel Method}

From [10], the conjugate morphological memories, $M_{\mathrm{YX}}=W_{\mathrm{XY}}^{*}$ and $W_{\mathrm{YX}}=$ $M_{\mathrm{XY}}^{*}$, also denoted by $W^{*}$ and $M^{*}$, perform the feedback scheme for bidirectional recall in a MBAM. The basic association mechanisms for perfect input in the $X$ to $Y$ direction are given by the following one-step procedure without thresholding

$$
\boldsymbol{x}^{\xi} \rightarrow\left\{W_{\mathrm{XY}} \mid M_{\mathrm{XY}}\right\} \rightarrow \boldsymbol{y}^{\xi} \rightarrow\left\{M_{\mathrm{YX}} \mid W_{\mathrm{YX}}\right\} \rightarrow \boldsymbol{x}^{\xi} .
$$



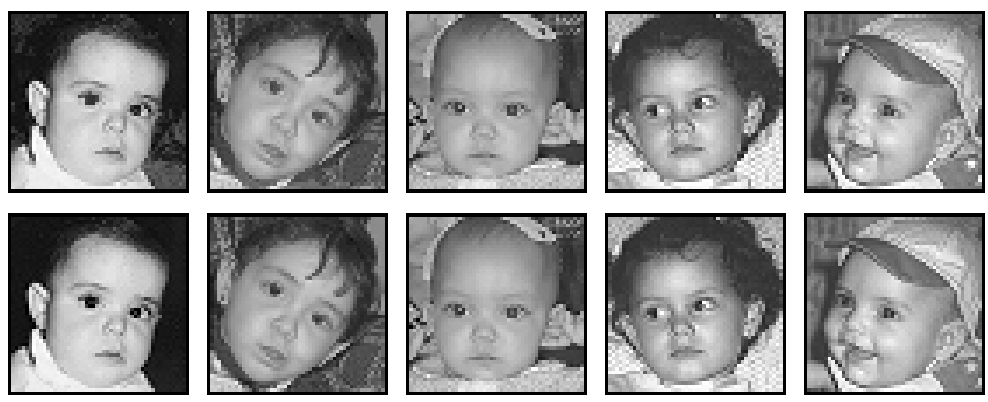

Fig. 2. The first row displays the associated $Y$ patterns as recalled by the memory $W_{\mathrm{XY}}$; the second row displays the associated $Y$ patterns as recalled by the memory $M_{\mathrm{XY}}$

Again, conditions for perfect recall in MBAMs must satisfy relations similar to (7) (for MAMs) in both the forward and feedback paths for the memories involved. Even if perfect recall can not be accomplished, MBAMs allow for heavy overlap of features as was demonstrated in [10] using boolean patterns.

We now turn our attention to noisy patterns. Let $I=\{1, \ldots, n\}$, a distorted version $\tilde{\boldsymbol{x}}^{\gamma}$ of the pattern $\boldsymbol{x}^{\gamma}$ has undergone an erosive change whenever $\tilde{\boldsymbol{x}}^{\gamma} \leq \boldsymbol{x}^{\gamma}$ or equivalently if $\forall i \in I, \tilde{x}_{i}^{\gamma} \leq x_{i}^{\gamma}$. A dilative change occurs whenever $\tilde{\boldsymbol{x}}^{\gamma} \geq \boldsymbol{x}^{\gamma}$ or equivalently if $\forall i \in I, \tilde{x}_{i}^{\gamma} \geq x_{i}^{\gamma}$. Let $L, G \subset I$ be two non-empty disjoint sets of indexes; if $\forall i \in L, \tilde{x}_{i}^{\gamma}<x_{i}^{\gamma}$ and $\forall i \in G, \tilde{x}_{i}^{\gamma}>x_{i}^{\gamma}$, then the distorted pattern $\tilde{\boldsymbol{x}}^{\gamma}$ is said to contain random noise. In order to deal efficiently with corrupted versions of exemplar patterns, the kernel method has proven to be useful in the binary case for MAMs 913] and MBAMs [10. Here, we will extend the kernel technique in MBAMs to store and recall non-boolean pattern associations.

The underlying idea of the kernel technique is to define a memory $M$ which associates with each input pattern $\boldsymbol{x}^{\gamma}$ an intermediate eroded pattern $\boldsymbol{z}^{\gamma}$ called the kernel pattern. Another associative memory $W$ is defined which associates each kernel pattern $\boldsymbol{z}^{\gamma}$ with the desired output pattern $\boldsymbol{y}^{\gamma}$. In terms of min-max sums, one obtains the equation, $W \nabla\left(M \nabla \boldsymbol{x}^{\gamma}\right)=\boldsymbol{y}^{\gamma}$. The combination of the two morphological memories $M$ and $W$ is what motivated the following definitions and results (proved in 12]); for application purposes we assume that pattern features are non-negative, i.e., $x_{i}^{\gamma} \geq 0$ for all $i, \gamma$.

Definition 4.1. Let $Z=\left(\boldsymbol{z}^{1}, \ldots, \boldsymbol{z}^{k}\right)$ be an $n \times k$ matrix. We say that $Z$ is a kernel for $(X, Y)$ with $X \neq Y$, if and only if $Z \neq X$ and there exists a memory $W$ such that $W \nabla\left(M_{\mathrm{ZZ}} \nabla \boldsymbol{x}^{\gamma}\right)=\boldsymbol{y}^{\gamma}$.

Definition 4.2. A set of patterns $Z \leq X$ is said to be a minimal representation of $X$ if and only if for $\gamma=1, \ldots, k, \boldsymbol{z}^{\gamma} \wedge \boldsymbol{z}^{\xi}=\mathbf{0} \forall \xi \neq \gamma, \boldsymbol{z}^{\gamma}$ contains at most one non-zero entry, and $W_{\mathrm{ZX}} \nabla \boldsymbol{z}^{\gamma}=\boldsymbol{x}^{\gamma}$.

Definition 4.3. A set of pattern vectors $X$ is said to be morphologically strongly independent (MSI) if and only if, $\forall \xi \neq \gamma$, the next two conditions are satisfied: 
1. $\forall \gamma \in\{1, \ldots, k\}, \boldsymbol{x}^{\gamma} \not \boldsymbol{x}^{\xi}$,

2. $\forall \gamma \in\{1, \ldots, k\}$, there $\exists j_{\gamma} \in\{1, \ldots, n\}$ such that

$$
x_{i}^{\gamma}-x_{i}^{\xi} \leq x_{j_{\gamma}}^{\gamma}-x_{j_{\gamma}}^{\xi}, \quad \forall i \in\{1, \ldots, n\} .
$$

Theorem 4.1. If $X$ is morphologically strongly independent, then there exists a set of patterns $Z \leq X$ with the property that for $\gamma \in\{1, \ldots, k\}$

1. $\forall \xi \neq \gamma, \boldsymbol{z}^{\gamma} \wedge \boldsymbol{z}^{\xi}=\mathbf{0}$

2. $\boldsymbol{z}^{\gamma}$ contains at most one non-zero entry, and

3. $W_{\mathrm{XX}} \nabla \boldsymbol{z}^{\gamma}=\boldsymbol{x}^{\gamma}$.

Corollary 4.1. If $X$ and $Z$ are as in Theorem 4.1, then $Z$ is a minimal representation of $X$.

Corollary 4.2. If $X$ and $Z$ are as in Theorem 4.1 and $W_{\mathrm{XY}}$ is a perfect associative recall memory, then $Z$ is a kernel for $(X, Y)$ with $W=W_{\mathrm{XY}} \nabla W_{\mathrm{XX}}$.

It is clear, from Corollary 4.2, that the recall mechanism in MBAMs is given by the following feed-forward network

$$
\boldsymbol{x}^{\xi} \rightarrow M_{\mathrm{ZZ}} \rightarrow W \rightarrow \boldsymbol{y}^{\xi} \rightarrow M_{\mathrm{VV}} \rightarrow W^{\prime} \rightarrow \boldsymbol{x}^{\xi},
$$

where, $W=W_{\mathrm{XY}} \nabla W_{\mathrm{XX}}, W^{\prime}=W_{\mathrm{YX}} \nabla W_{\mathrm{YY}}$, and $V$ is a kernel for $(Y, X)$. The conditions that, $W_{\mathrm{XX}} \nabla \boldsymbol{z}^{\gamma}=\boldsymbol{x}^{\gamma}$ and $W_{\mathrm{YY}} \nabla \boldsymbol{v}^{\gamma}=\boldsymbol{y}^{\gamma}$ are crucial for the recall capability of the memory scheme of (10) when presented with noisy inputs. Given a pair of minimal representations $Z, V$ which are also kernels, respectively, for $(X, Y)$ and $(Y, X)$, and a noisy version $\left(\tilde{\boldsymbol{x}}^{\gamma}, \tilde{\boldsymbol{y}}^{\gamma}\right)$ of the pattern association $\left(\boldsymbol{x}^{\gamma}, \boldsymbol{y}^{\gamma}\right)$ having the property that $\left(\boldsymbol{z}^{\gamma}, \boldsymbol{v}^{\gamma}\right) \leq\left(\tilde{\boldsymbol{x}}^{\gamma}, \tilde{\boldsymbol{y}}^{\gamma}\right)$ and $\left(M_{\mathrm{ZZ}} \nabla \tilde{\boldsymbol{x}}^{\gamma}, M_{\mathrm{VV}} \nabla \tilde{\boldsymbol{y}}^{\gamma}\right) \leq\left(\boldsymbol{x}^{\gamma}, \boldsymbol{y}^{\gamma}\right)$, then it must follow that

$$
W_{\mathrm{XX}} \nabla\left(M_{\mathrm{ZZ}} \nabla \tilde{\boldsymbol{x}}^{\gamma}\right)=\boldsymbol{x}^{\gamma} \text { and } W_{\mathrm{YY}} \nabla\left(M_{\mathrm{VV}} \nabla \tilde{\boldsymbol{y}}^{\gamma}\right)=\boldsymbol{y}^{\gamma} .
$$

Although the performance of the proposed feed-forward MBAM network when presented with noisy inputs can not be assured in a completely deterministic way, for any set $(X, Y)$ of $k$ associated patterns in $\mathbb{R}^{n} \times \mathbb{R}^{m}$, the expectation of recall capability is enhanced if $\min (n, m) \gg 0$ and $k \ll \min (n, m)$.

\section{Computation of Kernels}

From a theoretical point of view, Theorem 4.1 and its corollaries provide the foundation for the kernel method when applied to perfect inputs. In addition, the combined memory scheme suggested by (10) together with the kernel association shown in (11) provide a useful mechanism for bidirectional pattern recall of noisy inputs. On the other hand, it is clear that the condition of morphological strong independence of the sets $X$ and $Y$ will be rarely satisfied in practical situations and seems to be very restrictive in its possible applications. A practical solution to this dilemma is given by the following procedure. 


\section{Algorithm 5.1. [MBAM kernels by induced MSI.]}

STEP 1. Compute the global maximum $U$, and the global minimum $L$ of the input set $X$ that has $k$ patterns of dimension $n$, i.e., $U=\max (X)=\bigvee_{i=1}^{n} \bigvee_{\xi=1}^{k} x_{i}^{\xi}$ and $L=\min (X)=\bigwedge_{i=1}^{n} \bigwedge_{\xi=1}^{k} x_{i}^{\xi}$.

Step 2. Let $I=\{1, \ldots, n\}$. For $\xi=1, \ldots, k$, compute an index $i_{\xi} \in I$ where the first available maximum value occurs, i.e., let $x_{i_{\xi}}^{\xi}=\bigvee_{i \in I} x_{i}^{\xi}, I=I-\left\{i_{\xi}\right\}$, $\xi=\xi+1$, and recompute $x_{i_{\xi}}^{\xi}$ for the new pattern $\boldsymbol{x}^{\xi}$, hence $\forall \gamma \neq \xi, i_{\gamma} \neq i_{\xi}$.

STEP 3. Change the original pattern set $X$, at all positions $i_{\xi}$ for $\xi=1, \ldots, k$ with the $U$ and $L$ values determined in Step 1. Specifically, set $x_{i_{\gamma}}^{\xi}=U$ if $\gamma=\xi$ otherwise set it to $L$. It turns out, that the modified pattern set, denoted by $\hat{X}$, is a morphologically strongly independent set.

STEP 4. Apply to $\hat{X}$ the kernel method and the morphological memory scheme as described in Section 4 . The kernel $Z$ of $\hat{X}$ is readily obtained from Step 3, by defining for $i=1, \ldots, n$ and $\xi=1, \ldots, k, z_{i}^{\xi}=U$ if $i=i_{\xi}$ otherwise set it to 0 .

STeP 5. Repeat STeps 1-4 for set $Y$ to find the kernel $V$ of $\hat{Y}$. In this final step, a two way kernel $(Z, V)$ has been determined for $(X, Y)$.

To complete the description of Algorithm 5.1, we next prove that set $\hat{X}$ ( similarly for $\hat{Y}$ ) satisfies both conditions for MSI of Definition 4.3:

1. $\forall \gamma \in\{1, \ldots, k\}, x^{\gamma} \nless x^{\xi}$ for all $\xi \neq \gamma$; that is, an index $j_{\gamma} \in\{1, \ldots, n\}$ exists such that $x_{j_{\gamma}}^{\gamma}>x_{j_{\gamma}}^{\xi}$ for all $\xi \neq \gamma$. That this is true, follows immediately from the assignment made in STEP 3 by making the choice, $j_{\gamma}=i_{\gamma}$.

2. $\forall \gamma \in\{1, \ldots, k\}$, there is an index $j_{\gamma} \in\{1, \ldots, n\}$ such that, $\forall i \in\{1, \ldots, n\}$, $x_{j_{\gamma}}^{\gamma}-x_{j_{\gamma}}^{\xi} \geqslant x_{i}^{\gamma}-x_{i}^{\xi}$; take again, $j_{\gamma}=i_{\gamma}$, therefore

$U-L=\bigvee_{i=1}^{n}\left(x_{i}^{\gamma}-x_{i}^{\xi}\right) \geqslant x_{i}^{\gamma}-x_{i}^{\xi}$

Essentially, the kernel computation suggested in Algorithm 5.1, introduces an alternative MBAM scheme that substitutes (10), as follows,

$$
\begin{aligned}
& \boldsymbol{x}^{\xi} \rightarrow \hat{\boldsymbol{x}}^{\xi} \rightarrow M_{\mathrm{ZZ}} \rightarrow W_{\hat{\mathrm{X}} \hat{\mathrm{X}}} \rightarrow W_{\hat{\mathrm{X} Y}} \rightarrow \boldsymbol{y}^{\xi} \\
& \boldsymbol{x}^{\xi} \leftarrow W_{\hat{\mathrm{Y} X}} \leftarrow W_{\hat{\mathrm{Y}} \hat{\mathrm{Y}}} \leftarrow M_{\mathrm{VV}} \leftarrow \hat{\boldsymbol{y}}^{\xi} \leftarrow \boldsymbol{y}^{\xi},
\end{aligned}
$$

where, the recollection mechanism is based on the modified pattern sets $\hat{X}, \hat{Y}$ rather than the original $X, Y$ sets. Observe that induced MSI introduces a negligible amount of deterministic "artificial noise" to the original patterns which does not affect the MBAM performance if $\min (n, m) \gg 0$ and $k \ll \min (n, m)$. 


\section{Conclusion}

Several key steps have been achieved for enhancing the recall capability as well as the error correction rate of morphological associative memories in the case of boolean patterns. However, the kernel technique in the non-boolean case has required more elaborate concepts to deal effectively with corrupted inputs. Morphological strong independence is a sufficient condition for building minimal representations and kernels; however, in most practical applications, the association pattern sets may not satisfy this requirement. Therefore, at the expense of reducing storage capacity, the induced MSI procedure presented here is useful for generating a two way kernel in a MBAM that is quite robust to random noise for arbitrary non-boolean associations.

\section{References}

1. Chen H. et al.: Higher order correlation model for associative memories. Neural Networks for Computing, J. S. Denker, Ed. 151 (1986) AIP Proc.

2. Cuninghame-Green, R.: Minimax algebra. Lectures Notes in Economics and Mathematical Systems 166 (1979), New York, Springer.

3. Hopfield, J. J.: Neurons with graded response have collective computational properties like those of two state neurons. Proc. of the National Academy of Sciences 81 (1984) 3088-3092, U.S.A.

4. Kohonen, T.: Correlation matrix memory. IEEE Trans. Computers C-21 (1972) 353-359.

5. Kosko, B.: Bidirectional associative memories. IEEE Trans. Systems, Man, and Cybernetics 18(1)(1988) 49-60.

6. McEliece, R. et al.: The capacity of the Hopfield associative memory. Trans. Information Theory 1 (1987) 33-45.

7. Okajima, K., Tanaka, S., Fujiwara, S.: A heteroassociative memory with feedback connection. Proc. IEEE 1st Inter. Conf. Neural Networks 2 (1987) 711-718.

8. Ritter, G. X.: Image Algebra. Unpublished manuscript, available at ftp://ftp.cise.ufl.edu/pub/scr/ia/documents (1994) University of Florida, Gainesville, FL.

9. Ritter, G. X., Sussner, P., Diaz de Leon, J. L.: Morphological associative memories. IEEE Trans. Neural Networks 9(2) (1998) 281-293.

10. Ritter, G. X., Diaz de Leon, J. L., Sussner, P.: Morphological bidirectional associative memories. Neural Networks 12(6) (1999) 851-867.

11. Ritter, G. X., Urcid, G.: Minimal representations and morphological associative memories for pattern recall. Proc. 7th Iberoamerican Congress on Pattern Recogniton (2002).

12. Ritter, G. X., Urcid, G., Iancu, L.: Reconstruction of noisy patterns using morphological associative memories. J. of Math. Imaging and Vision 19(5) (2003) 95-111.

13. Sussner, P.: Observations on morphological associative memories and the kernel method. Neurocomputing 31 (2000) 167-183.

14. Sussner, P.: Binary autoassociative morphological memories based on the kernel and dual kernel methods. Int. Joint Conf. on Neural Networks (2003).

15. Wu, Y., Pados, D. A.: A feedforward bidirectional associative memory. IEEE Trans. Neural Networks 11(4) (2000) 859-866. 